\title{
Renal cell carcinoma metastasis in the bladder diagnosed based on transurethral resection after hematuria: a case report
}

\begin{abstract}
Introduction: Despite the population's greater access to imaging tests and consequen diagnosis of renal cancer in early stages, we continue to encounter neoplasms in locally advanced and metastatic stages.

Case report: We report a patient admitted to the emergency department of the Hospital Geral de Fortaleza with hematuria and urinary retention. After the patient underwent computed tomography and transurethral resection, a diagnosis of renal cell carcinoma metastasis in the bladder was confirmed based on immune histo chemistry results.
\end{abstract}

Discussion: Metastasis of renal cell carcinoma in the bladder is rare, with few cases reported in the literature on synchronic presentation.

Final considerations: Although rare, we must consider the possibility of metastasis of renal cell carcinoma in patients who present with hematuria, especially in those previously diagnosed with renal cancer.
Volume 10 Issue 6 - 2020

Lucas De Oliveira Lima,Tadeu J F L Campos, Francisco E DeV Filho, André C M Lima,Ana C M De Queiroz, Marcos F H Rocha

Department of Urology, Hospital Geral De Fortaleza, Brazil

Correspondence: Lucas de Oliveira Lima, Department of Urology, Rua Luciano Magalhães, 333, Brazil, Email lucasliaufc@gmail.com

Received: December II, 2020 | Published: December 18 2020

Keywords: renal cancer, metastasis, bladder, hematuria

\section{Introduction}

The incidence of renal cancer has increased in recent decades as a result of greater access to imaging tests among the general population. In parallel to this increase in incidence, there is a clear reduction in the aggressiveness of tumors at the time of diagnosis. ${ }^{1,2}$

Despite this change in the presentation pattern of renal tumors, we continue to encounter patients being diagnosed with tumors in advanced stages due to local or systemic complications of locally advanced and/or metastatic disease.

Kidney cancer has a predilection for hematogenous spread to the liver and lungs. Metastasis to other sites is rarer, including the bones and brain. In this article, we describe a rare case of metastasis of kidney cancer to the bladder, with a diagnosis being confirmed based on transurethral resection of the bladder mass after frank hematuria. ${ }^{3}$

\section{Case presentation}

In June 2020, a 52-year-old woman presented with complaints of frank hematuria, dysuria, and intermittent episodes of acute urinary retention. She also reported hyporexia, adynamia, weight loss, and severe pain in the hypogastrium and lower limbs.

On July 21, 2020, she underwent outpatient transvaginal ultrasonography that revealed a lesion suspected to be bladder cancer. Thus, after a few days of persistent hematuria, she was referred to the emergency department of the Hospital Geral de Fortaleza, where she was admitted on August 3, 2020.

On admission, she complained of hematuria, pain in the hypogastrium, and a sensation of incomplete bladder emptying. She underwent serum tests that showed anemia with a hemoglobin count of $8.5 \mathrm{~g} / \mathrm{dL}$, as the only relevant finding.
The following day, she underwent contrast-enhanced computed tomography of the abdomen, pelvis, and chest. The chest image showed only nonspecific findings of probable infectious scarring.

The abdominal image showed enlargement of the left kidney with a solid, expansive lesion that exhibited irregular contours and heterogeneous contrast enhancement, measuring $6.4 \mathrm{~cm}$ along its longest axis, with involvement of the ureter and gonadal vein. In the pelvis, an extensive, expansive formation was identified in the bladder with a vegetative and infiltrative aspect, with uptake similar to that associated with renal injury and signs of invasion of the abdominal wall and pubic bones (Figures 1,2).

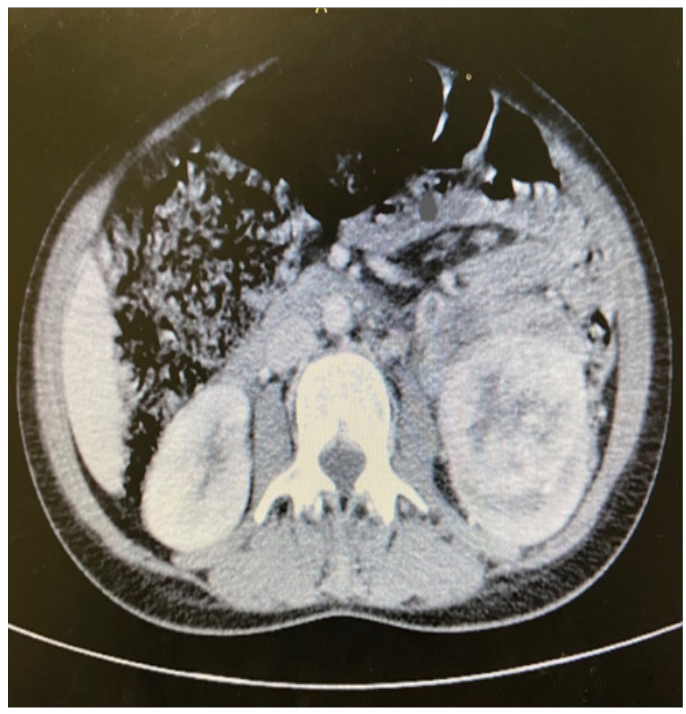

Figure I Computed tomography image of a massive solid lesion in the left kidney. 


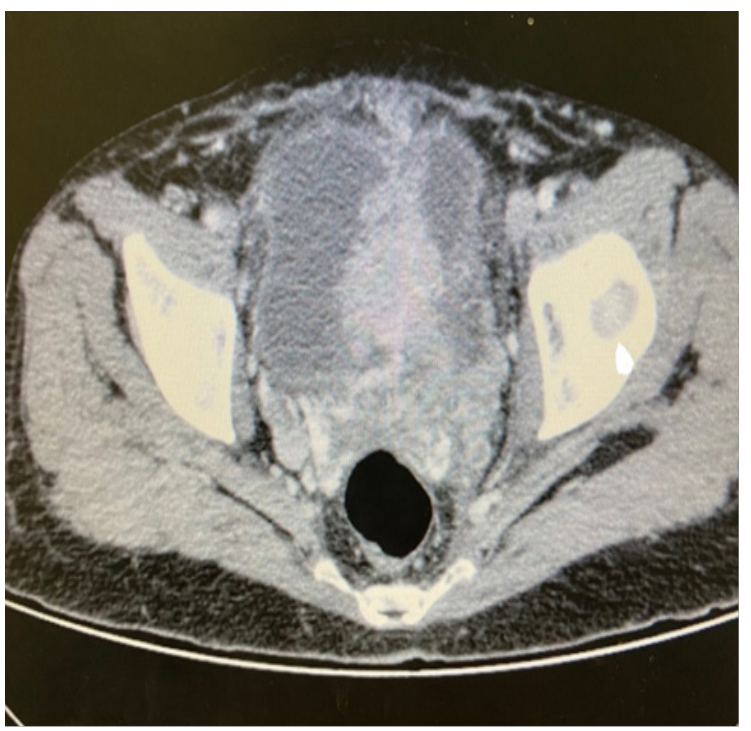

Figure 2 Computed tomography image of an infiltrative mass in the bladder.

On August 11, 2020, the patient underwent cystoscopy with partial transurethral resection of a large bladder lesion with an aspect not suggestive of a urothelial tumor. Histopathological findings of the resected specimen suggested an invasive clear cell carcinoma, and an immune histochemical study was planned.

Immuno histochemical evaluation showed a clear cell carcinoma without the expression of cytokeratin (CK)7 and CK20, in addition to diffuse positivity for PAX8, suggesting metastasis of the primary renal site.

Considering a metastatic kidney tumor, the patient was classified based on the criteria of the Memorial Sloan-Kettering Cancer Center Score for Metastatic Renal Cell Carcinoma as high risk, wherein there is no benefit of cytoreductive nephrectomy.

The patient was then discharged on September 2, 2020, for outpatient follow-up with an oncologist, palliative care physician, and radiotherapist. He underwent new computed tomography of the abdomen and pelvis in late September 2020, which demonstrated an extension of the renal injury to the ileopsoas muscle and the presence of a thrombus in the left renal vein, in addition to multiple retroperitoneal lymph nodes.

The patient was referred for palliative anti-pain radiotherapy in the pelvis, ending on October 27, 2020. In November 2020, systemic therapy with sunitinib was initiated. The patient currently remains on an outpatient basis, undergoing systemic treatment and palliative care for pain control.

\section{Discussion}

Renal neoplasia is currently diagnosed in increasingly early stages in the form of incident alomas in asymptomatic patients who undergo routine imaging tests. The proportion of patients with locally advanced or metastatic disease at the time of diagnosis has been decreasing. ${ }^{1}$

The hematogenous spread of kidney cancer occurs more frequently in the liver and lungs. The involvement of other organs occurs to a lesser extent, including the bones and brain. Rare sites of metastasis include the thyroid, pancreas, spleen, and skin, among others. ${ }^{3}$

Bladder metastasis represents less than $2 \%$ of all tumors in this organ. Metastasis of renal tumors to the bladder occurs very rarely.
This metastatic involvement of the bladder may be metachronic or synchronous, with the latter being rarer.

In most cases of bladder metastasis, the diagnosis of kidney tumors is already well established, with rare cases such as the present case in which the diagnosis was motivated by hematuria from bladder metastasis. It occurs more commonly in patients with multiple metastases in other organs and confers poor prognosis. ${ }^{5}$

Kruck et al., ${ }^{6}$ reported a similar case to this one in 2013, where the synchronous diagnosis of bladder metastasis and renal tumor was motivated by a hematuria. In the literature there are few cases reported in the synchronic scenario, as in this case. ${ }^{6-8}$

For these patients, treatment should be based on risk stratification of metastatic kidney tumors through scores, such as the Memorial Sloan-Kettering Cancer Center Score for Metastatic Renal Cell Carcinoma. Depending on a patient's classification, cytoreductive nephrectomy may or may not be indicated, in addition to systemic therapy with tyrosine kinase inhibitors or immunotherapy. ${ }^{9}$

Bladder metastasis can be treated with transurethral resection or partial cystectomy, in addition to systemic treatment. In this case presented, the resection was only partial and cystectomy was contraindicated by the patient's poor status, being submitted only to systemic and palliative treatment. ${ }^{10}$

Although rare, it is worth noting the possibility of metastatic bladder involvement in kidney cancer as a cause of hematuria in patients with advanced kidney disease. In the present case, the differential diagnosis between an advanced renal cell tumor with metastasis in the bladder of a synchronic urothelial tumor of the renal pelvis and bladder was important, since these pathologies correspond to different systemic treatment proposals.

\section{Conclusions}

Despite the popularization of imaging tests and consequent diagnosis of renal tumors in early stages, we continue to encounter diagnoses in very advanced stages of the disease, as in this case, in which the patient in question, in addition to local extension of the tumor, presented with synchronous metastasis in the bladder evolving with gross hematuria.

Therefore, it is important to remember the possibility of metastasis in the bladder in patients with renal tumor that evolves with hematuria.

\section{Conflicts of interest}

The author declares no conflict of interest.

\section{Acknowledgments}

None.

\section{Funding}

None.

\section{References}

1. Chow WH, Devesa SS, Warren JL, Fraumeni JF. Rising incidence of renal cell cancer in the United States. JAMA. 1999;281(17):1628-1631.

2. Decastro GJ, McKiernan JM. Epidemiology, clinical staging, and presentation of renal cell carcinoma. Urol Clin North Am. 2008;35(4):581592.

3. Cronin RE, Kaehny WD, Miller PD, et al. Renal cell carcinoma: Unusual systemic manifestations. Medicine (Baltimore). 1976;55(4):291-311 
4. Gelister JS, Falzon M, Crawford R, Chapple CR, Hendry WF. Urinary tract metastasis from renal carcinoma. Br J Urol. 1992;69(3):250-252

5. Ebele JN, Sauter G, Epstein JI, editors. World Health Organization Classification of Tumours. Pathology and Genetics of Tumours of the Urinary System and Male Genital Organs; IARC, Lyon: 2004. Clear Cell Renal Carcinoma. p. 23-25

6. Kruck S, Scharpf M, Stenzl A, et al. A rare case of synchronous renal cell carcinoma of the bladder presenting with gross hematuria. Rare Tumors. 2013;5(2):72-74

7. Tsai TH, Tang SH, Chuang FP, et al. Ipsilateral synchronous neoplasms of kidney presenting as acute pyelonephritis and bladder metastasis. Urology. 2009;73(5):1163.
8. Uygur MC, Ozen HA, Sungur A, et al. A solitary and synchronous metastasis of renal cell carcinoma to the bladder. Int Urol Nephrol.1994;26(5):529-33.

9. EAU Guidelines. Edn. presented at the EAU Annual Congress Amsterdam. ISBN. 2020.

10. Dogra P, Kumar A, Singh A. An unusual case of Von HippleLindau (VHL) syndrome with bilateral mutlicentric renal cell carcinoma with synchronous solitary urinary bladder metastasis.Int Urol Nephrol. 2007;39:11-14. 